\title{
Nitrogenase activity in tropical carbonate marine sediments ${ }^{*}$
}

\author{
Judith M. O'Neil ${ }^{1, * *}$, Douglas G. Capone ${ }^{2}$ \\ ${ }^{1}$ Marine Sciences Research Center, State University of New York, Stony Brook, New York 11794-5000, USA \\ ${ }^{2}$ Chesapeake Biological Laboratory, Center for Environmental and Estuarine Studies, University of Maryland, Solomons, \\ Maryland 20688-0038, USA
}

\begin{abstract}
Rates of nitrogenase activity were investigated in carbonate sediments of shallow tropical waters using the acetylene $\left(\mathrm{C}_{2} \mathrm{H}_{2}\right)$ reduction method. Nitrogenase activity was significantly higher in fine grained sediments than in coarser grained sands. The importance of oxygen, ammonium $\left(\mathrm{NH}_{4}^{+}\right)$and organic substrate availability in regulating nitrogenase activity in these sediments was evaluated. The effect of $\mathrm{O}_{2}$ was variable and appeared to be influenced by physical disturbance of the sediment. Ammonium did not appear to inhibit rates of $\mathrm{N}_{2}$ fixation over the range of in situ concentrations observed, as there was no significant correlation of $\mathrm{N}_{2}$ fixation rates and pore water $\mathrm{NH}_{4}^{+}$. Neither additions of $\mathrm{NH}_{4}^{+}$nor MSX (L-methionine-DL-sulfoximine, a nitrogenase derepressor) had any consistent effect on $\mathrm{N}_{2}$ fixation. $\mathrm{N}_{2}$ fixation rates were correlated with total organic content of sediments. Additions of mannitol and glucose led to significant, concentration-dependent stimulation of $\mathrm{N}_{2}$ fixation. We therefore conclude that organic substrate availability is a major factor controlling rates of nitrogenase activity in these shallow carbonate sediments. Based on our experiments, benthic heterotrophic bacteria in carbonate sediments could account for daily $\mathrm{N}_{2}$ fixation inputs of 0.07 to $5.2 \mathrm{mg} \mathrm{N} \mathrm{m}^{-2}(0.03$ to $1.9 \mathrm{~g} \mathrm{~N} \mathrm{~m}^{-2} \mathrm{yr}^{-1}$ ). These estimates indicate that the extensive areas of carbonate sediments in these environments may contribute a substantial amount of nitrogen to shallow tropical and subtropical ecosystems.
\end{abstract}

\section{INTRODUCTION}

Shallow tropical ecosystems such as coral reefs and seagrass beds are notable for sustaining intense biological production despite low ambient concentrations of nutrients. Nitrogen has generally been considered the primary limiting nutrient in shallow tropical environments (Patriquin \& Knowles 1972, D'Elia \& Wiebe in press; cf. Smith 1984, Short et al. 1985). Accordingly, much research has focused on the primary input of nitrogen through biological fixation in shallow tropical environments. Nitrogen-fixing organisms in these areas include: cyanobacteria that occur as mats and epiphytes (Capone 1983, 1988); heterotrophic bacteria in coral rubble (Crossland \& Barnes 1976, Hanson \& Gundersen 1976), and sediments of the seagrass rhizosphere (Capone 1982). The extensive carbonate sediments surrounding coral reefs and seagrass

\footnotetext{
- Contribution No. 646 of the Marine Sciences Research Center, and No. 1220 of the Bermuda Biological Station

- Present address: Chesapeake Biological Laboratory
}

beds have also been implicated as sites of heterotrophic $\mathrm{N}_{2}$ fixation (Patriquin \& Knowles 1975, Hanson \& Gundersen 1976, Corredor \& Capone 1985). Heterotrophic bacterial $\mathrm{N}_{2}$ fixation has generally been considered minor when compared to autotrophic cyanobacterial $\mathrm{N}_{2}$ fixation in reef environments (Hanson \& Gundersen 1977. Howarth et al. 1988). However, there is little information on the quantitative importance of new nitrogen inputs occurring within the sedimentary environment, or on the physiology of naturally occurring heterotrophic $\mathrm{N}_{2}$ fixing organisms.

Oxygen, ammonium and organic substrate concentration and supply are important determinants of $\mathrm{N}_{2}$ fixation in sediments (Capone 1983, 1988). Sediments often have low $\mathrm{O}_{2}$ levels and high concentrations of organic carbon and, therefore, are potentially suitable sites for heterotrophic $\mathrm{N}_{2}$ fixation. However, shallow sediments commonly have high $\mathrm{NH}_{4}^{+}$levels as well, which can inhibit $\mathrm{N}_{2}$ fixation.

Our objectives were to obtain rates of benthic, heterotrophic bacterial $\mathrm{N}_{2}$ fixation in a variety of carbonate sediments, and to examine factors that regulate this 
process in situ. Estimates of in situ nitrogen fixation were determined in coarse and fine grained sediments from geographically diverse locations using the acetylene reduction assay. Rates of nitrogen fixation in sediments experimentally manipulated with $\mathrm{O}_{2}, \mathrm{NH}_{4}^{+}$and organic substrates were also obtained. In situ repression of nitrogenase activity by $\mathrm{NH}_{4}^{+}$was also investigated using additions of L-methionine-DL-sulfoximine (MSX).

\section{METHODS}

Study sites. Nitrogenase activity was investigated in shallow (1 to $25 \mathrm{~m}$ water depth) carbonate sediments of: Bermuda and San Salvador Island, Bahamas (Fig. 1A, B), along the southwest coast of Puerto Rico (Fig. 1C), and at several reef sites along the central portion of the Great Barrier Reef, Australia (Fig. 1D). Sediments at the sites fell into 2 broad categories: muds and very fine grained sands (phi $>2$ ), or fine to coarse grained sands $(\mathrm{phi}<2$ ). Experiments in Puerto Rico were conducted in March and October 1984. Studies in Bermuda were carried out during July and August 1985. Work in Australia took place in November and December 1985. Experiments in San Salvador took place during July 1986 and January-February 1987.

Sediment cores. Cores of the top 1 to $5 \mathrm{~cm}$ of sediment were taken by SCUBA or snorkeling, with plastic core tubes (cut off $30 \mathrm{cc}$ syringes, $10 \mathrm{~cm}$ length $\times 2 \mathrm{~cm}$ diam.). Previous studies found that the majority of nitrogenase activity occurs in the top few cm of sediments (Corredor \& Capone 1985). Samples were returned to the laboratory and processed as soon after sampling as possible (within $24 \mathrm{~h}$ ). Sediment cores were sectioned in 2 to $5 \mathrm{~cm}$ increments and extruded directly (with neither homogenization nor stirring) into experimental flasks. For certain experiments, sediments were homogenized manually with an approximately equal volume of filtered seawater to reduce variability. An intial experiment compared nitrogenase activity in mechanically (Waring blender, low speed) homogenized samples and non-homogenized samples. Flasks were sealed with gas-tight rubber stoppers. Deoxygenated, $0.45 \mu \mathrm{m}$ filtered seawater was added in those experiments requiring a liquid phase for amendments. For anaerobic experiments, stoppered flasks were gassed with $\mathrm{N}_{2}$ for 2 to $3 \mathrm{~min}$ through incurrent and excurrent hypodermic needles inserted through the stopper. The headspaces of the flasks were occasionally sampled and checked for $\mathrm{O}_{2}$ concentrations using electron capture gas chromatography.

Acetylene reduction assay. Nitrogenase activity was measured using the acetylene reduction assay (Stewart et al. 1967, Hardy et al. 1968). Acetylene $\left(\mathrm{C}_{2} \mathrm{H}_{2}\right)$, generated from calcium carbide, was added to the experi-

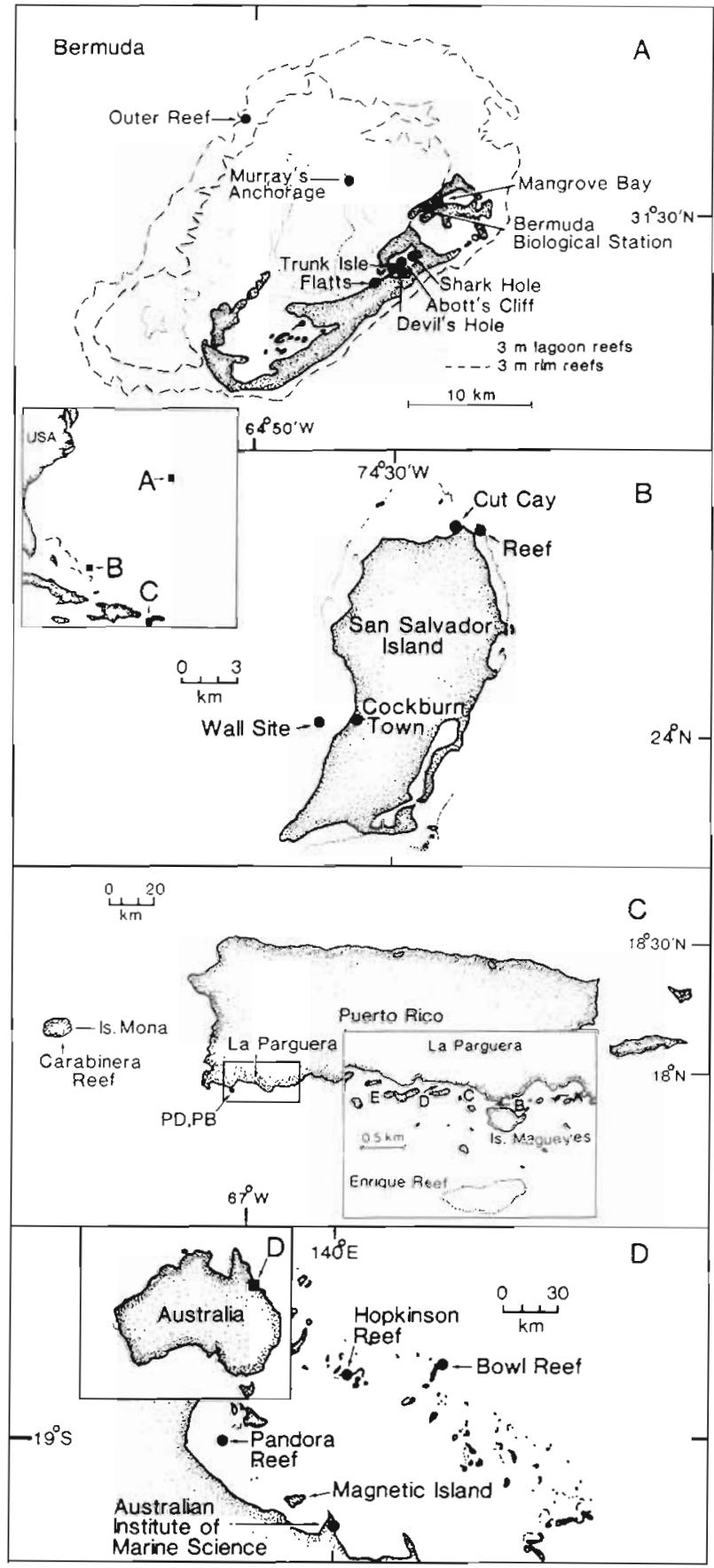

Fig. 1. Study sites. (A) Bermuda; (B) San Salvador Island, Bahamas; (C) La Parguera, Puerto Rico; (D) Great Barrier Reef, Australia

mental flasks to a final volume equal to $20 \%$ of the gas head space volume (vol : vol). Flasks were incubated in the dark and experiments were carried out within $2 \mathrm{C}^{\circ}$ of ambient temperature $\left(25\right.$ to $\left.30^{\circ} \mathrm{C}\right)$. Control experiments with sediment and no additions of $\mathrm{C}_{2} \mathrm{H}_{2}$, as well as flasks containing filtered seawater alone with additions of $\mathrm{C}_{2} \mathrm{H}_{2}$, were also conducted. 
Gas samples $(100 \mu l)$ were withdrawn from the headspace of the experimental flasks with a gas-tight syringe and analyzed immediately for ethylene $\left(\mathrm{C}_{2} \mathrm{H}_{4}\right)$ production by flame ionization in a Shimadzu Mini 2 gas chromatograph. Gases were separated on a $2 \mathrm{~m} \times$ $3 \mathrm{~mm}$ Porapak R column, held at $70^{\circ} \mathrm{C}$. Injector temperature was $150^{\circ} \mathrm{C}_{i} \mathrm{~N}_{2}$ carrier flow was ca $30 \mathrm{ml} \mathrm{min}{ }^{-1}$; $\mathrm{H}_{2}$ was ca $30 \mathrm{ml} \mathrm{min}^{-1}$; air flow was ca $300 \mathrm{ml} \mathrm{min}^{-1}$.

Peak heights of $\mathrm{C}_{2} \mathrm{H}_{4}$ were measured on a HewlettPackard $3390 \mathrm{~A}$ integrator and converted to $\mathrm{nmol} \mathrm{C}_{2} \mathrm{H}_{4}$ by comparison to peaks of $\mathrm{C}_{2} \mathrm{H}_{4}$ standards of known concentrations. Sediment samples were dried to constant weight at 60 to $100^{\circ} \mathrm{C}$, and rates of $\mathrm{C}_{2} \mathrm{H}_{4}$ production were normalized to dry weight of sediment.

Rates of $\mathrm{C}_{2} \mathrm{H}_{2}$ reduction (nmol $\mathrm{C}_{2} \mathrm{H}_{4}$ [g dry sed.] ${ }^{-1}$ $\mathrm{h}^{-1}$ ) were determined by performing linear regression analysis to determine the average slope of replicates for each parameter over the initial $24 \mathrm{~h}$ of incubation. Standard errors and single classification ANOVAs (Sokal \& Rohlf 1981) were also determined.

Assay amendments. The effects of $\mathrm{O}_{2}, \mathrm{NH}_{4}^{+}$and organic substrate availability on rates of $\mathrm{C}_{2} \mathrm{H}_{2}$ reduction were investigated by amending sediments with varying concentrations of each compound being investigated.

To determine the effects of $\mathrm{O}_{2}$, samples were either incubated aerobically $\left(20 \% \mathrm{O}_{2}\right.$, i.e. air), or anaerobically by flushing with $\mathrm{O}_{2}$-free $\mathrm{N}_{2}$. The effects of $\mathrm{NH}_{4}^{+}$ were investigated using additions of $\mathrm{NH}_{4} \mathrm{Cl}(100 \mu \mathrm{M}$ to $5 \mathrm{~m} M$ to flasks by syringe injection through the gastight stoppers.

The extent of in situ repression of nitrogenase activity by $\mathrm{NH}_{4}^{+}$was also investigated using additions of MSX. MSX is an inhibitor of glutamine synthetase, and its addition can cause the derepression of the nitrogenase enzyme in the presence of $\mathrm{NH}_{4}^{+}$(Gordon \& Brill 1974). Stimulation of nitrogenase activity in the presence of MSX would suggest that in situ $\mathrm{N}_{2}$ fixation is occurring at levels below full potential due to repression by $\mathrm{NH}_{4}^{+}$(Yoch \& Whiting 1986, Capone 1988).

The effects of organic substrate availability on nitrogenase activity were investigated by amending sediments with readily oxidizable organic substrates (10 $\mu M$ to $3 \mathrm{mM}$ glucose and mannitol).

Porewater analysis. Sediments were analyzed for porewater $\mathrm{NH}_{4}^{+}$using standard seawater methods (Strickland \& Parsons 1972). Manual wet chemical methods were used in Bermuda and Australia, while a Technicon Auto Analyzer was available for the studies in San Salvador and Puerto Rico. Porewater from sites in Bermuda and Puerto Rico was sampled using a 'vampire stake' or spear sampler, which withdrew porewater from a given depth using a syringe. Porewater from sites in San Salvador and Australia was obtained by filtering intact sections of cores and collecting the filtrate.
Organic content of sediments. The amount of organic matter in sediments, expressed as \% total weight of the sediments, was determined by calculating the difference between the dry weight of a given volume of sediment and the weight after combustion at $450^{\circ} \mathrm{C}$ for $24 \mathrm{~h}$. This method does not account for loss of some inorganic carbon and, therefore, may result in a slight overestimate of total organic content (Gaudette et al. 1974).

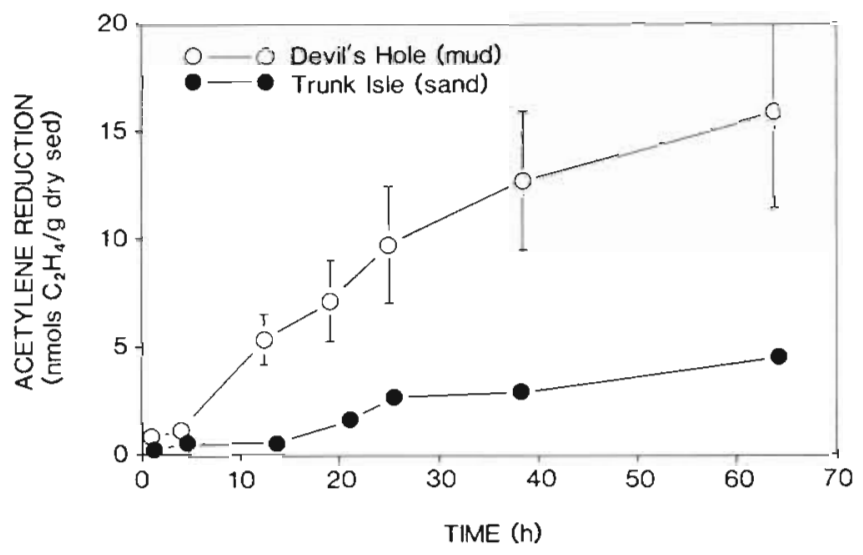

Fig. 2. Time course of nitrogenase activity (acetylene reduction) in muddy and sandy sediment from Harrington Sound, Bermuda. Error bars indicate \pm standard error; $\mathrm{n}=3$

\section{RESULTS}

Nitrogenase activity, while often low, was detected in all sediments examined. Ethylene production was generally evident within the first 1 to $2 \mathrm{~h}$ of each experiment and remained linear for up to $60 \mathrm{~h}$ (Fig. 2); rates have been expressed for the initial 12 to $24 \mathrm{~h}$ of assay. Among the sediments examined, rates were significantly higher $(\mathrm{p} \ll 0.001)$ in finer grained muds (Table 1; Fig. 2), compared to medium and coarser grained sands (Table 2; Fig. 2). For similarly sized sediments in Bermuda, where the broadest comparison was performed, rates of nitrogenase activity were significantly higher $(\mathrm{p}<0.005)$ at inshore sites than offshore (Fig. 1 $\mathrm{A}_{\text {; }}$ Tables 1 and 2). Among the muds sampled, highest rates of $\mathrm{C}_{2} \mathrm{H}_{2}$ reduction were found in the eutrophic sediments of La Parguera Channel, Puerto Rico (0.9 to 1.7 mmol $\mathrm{C}_{2} \mathrm{H}_{4}$ [g dry sed.] ${ }^{-1} \mathrm{~h}^{-1}$ ) (Table 1). Muddy sediments from a eutrophic, nearshore reef of the central Great Barrier Reef (Pandora Reef) had nitrogenase activity comparable to similar sediments around Bermuda. Rates of activity of fine through coarse grained sands were, for the most part, similar among all environments examined, ranging from 0.04 to $0.29 \mathrm{nmol} \mathrm{C}_{2} \mathrm{H}_{4}$ (g dry wt) ${ }^{-1} \mathrm{~h}^{-1}$ (Table 2).

Control experiments showed no ethylene production in samples of filtered seawater plus $\mathrm{C}_{2} \mathrm{H}_{2}$, nor in flasks 
Table 1. Average nitrogenase activity in fine grained carbonate sediments \pm standard error between days (no. of experiments on different days)

\begin{tabular}{|c|c|c|c|c|c|c|}
\hline Site & $\begin{array}{c}\text { Nitrogenase activity } \\
\text { (nmol }(g \text { dry sed. }]^{-1} \mathrm{~h}^{-1} \text { ) }\end{array}$ & $\begin{array}{l}\text { Anmonium } \\
\qquad(\mu M)\end{array}$ & $\begin{array}{c}\text { Organic content } \\
(\%)\end{array}$ & Porosity & $\begin{array}{l}\text { Water depth } \\
(\mathrm{m})\end{array}$ & $\begin{array}{l}\text { Sediment } \\
\text { type }\end{array}$ \\
\hline \multicolumn{7}{|l|}{ Bermuda } \\
\hline Mangrove Bay & $0.63 \pm 0.36(3)$ & 31 & 6.8 & - & 1 & Silty sand \\
\hline Devils Hole & 0.36 & 37 & 5.5 & 0.83 & 28 & Fine mud \\
\hline Abott's Cliff & 0.36 & 21 & 4.3 & 0.78 & 17 & Mud \\
\hline Shark Hole & 0.31 & - & 2.0 & 0.61 & 4 & Fine sand \\
\hline \multicolumn{7}{|l|}{ Puerto Rico } \\
\hline \multicolumn{7}{|c|}{ LaParguera Channel } \\
\hline D1 & 1.63 & 78 & 2.3 & - & $4-5$ & Fine mud \\
\hline D2 & $1.20 \pm 0.12$ & 529 & 2.9 & 0.70 & $4-5$ & Fine mud \\
\hline D3 & 1.43 & 104 & 2.1 & - & $4-5$ & Fine mud \\
\hline B1 & 0.95 & 244 & 1.6 & - & $4-5$ & Fine mud \\
\hline B3 & 1.71 & 410 & 2.6 & - & $4-5$ & Fine mud \\
\hline \multicolumn{7}{|l|}{ Australia } \\
\hline Pandora Reef & 0.44 & 35 & 1.6 & 0.58 & 5 & Mud \\
\hline
\end{tabular}

Table 2. Average nitrogenase activity in coarse grained carbonate sediments \pm standard error between sampling days (no. of experiments)

\begin{tabular}{|c|c|c|c|c|c|c|}
\hline Site & $\begin{array}{l}\text { Nitrogenase activity } \\
\text { (nmol [g dry sed. }]^{-1} \mathrm{~h}^{-1} \text { ) }\end{array}$ & $\underset{(\mu M)}{\text { Ammonium }}$ & $\begin{array}{c}\text { Organic } \\
\text { content } \\
(\%)\end{array}$ & Porosity & $\begin{array}{c}\text { Water } \\
\text { depth } \\
\text { (m) }\end{array}$ & Sediment type \\
\hline \multicolumn{7}{|l|}{ Bermuda } \\
\hline Trunk Island & 0.13 & 27 & 1.4 & 0.61 & 7 & Medium sand \\
\hline Flatt's Inlet & $0.06 \pm 0.02$ & - & 2.0 & 0.61 & 1 & Medium sand \\
\hline Outer Reef & $0.23 \pm 0.18(4)$ & 3 & 1.1 & 0.64 & 13 & Coarse sand \\
\hline Murray's Anchorage & 0.19 & - & 2.3 & 0.71 & 23 & Fine/med. mix sand \\
\hline \multicolumn{7}{|l|}{ San Salvador } \\
\hline Wall & 0.09 & 107 & 1.9 & - & 20 & Fine sand \\
\hline Cut Cay & $0.08 \pm 0.01(6)$ & - & - & - & 3 & Medium sand \\
\hline Reef & $0.04 \pm 0.03(2)$ & 6.4 & 3.0 & - & 5 & Coarse sand \\
\hline \multicolumn{7}{|l|}{ Puerto Rico } \\
\hline $\mathrm{B} 2$ & $0.18 \pm 0.01$ & 57 & 2.4 & 0.55 & 5 & Medium sand \\
\hline Enrique Reef & 0.15 & - & - & 0.30 & 5 & Coarse sand \\
\hline Carabinero Reef, \#1 & 0.14 & 6.2 & - & - & 15 & Medium sand \\
\hline Carabinero Reef, \#2 & $0.25 \pm 0.04(2)$ & 13 & - & - & 15 & Medium sand \\
\hline \multicolumn{7}{|l|}{ Australia } \\
\hline Hopkinson Reef, A & $0.14 \pm 0.005(9)$ & 12 & 0.69 & 0.36 & 20 & Fine sand (backreef) \\
\hline Hopkinson Reef, B & 0.08 & 4.1 & 0.66 & 0.39 & 7 & Medium sand (backreef) \\
\hline Hopkinson Reef, C & 0.04 & 4.5 & 0.72 & - & 2 & Coarse sand (reef flat) \\
\hline Hopkinson Reef, D & $0.16 \pm 0.07(2)$ & 6.1 & - & - & 20 & Medium sand (forereef) \\
\hline Bowl Reef, A & $0.09 \pm 0.02(2)$ & 22 & - & - & 10 & Fine sand (backreef) \\
\hline Bowl Reef, B & 0.14 & - & - & - & 20 & Medium sand (backreef) \\
\hline Bowl Reef, C & 0.28 & 1 & - & - & 10 & Coarse sand (backreef) \\
\hline
\end{tabular}

with sediment without $\mathrm{C}_{2} \mathrm{H}_{2}$. There was no difference in rates between light and dark incubations, suggesting that the majority of the activity in sediments was heterotrophic, rather than light-dependent cyanobacterial or photosynthetic bacterial $\mathrm{N}_{2}$ fixation (data not shown).

The depth distribution of nitrogenase activity varied qualitatively between fine and coarse grained sediments in Puerto Rico incubated under $\mathrm{N}_{2}$. Rates of activity decreased with depth in sandy sediment at Site $\mathrm{B} 2$, nearing zero at $10 \mathrm{~cm}$, while nitrogenase activity downcore in finer sediment at Site D2 still maintained relatively high rates to a depth of 8 to $10 \mathrm{~cm}$ (Fig. 3).

Initial experiments in Bermuda compared 2 methods of 


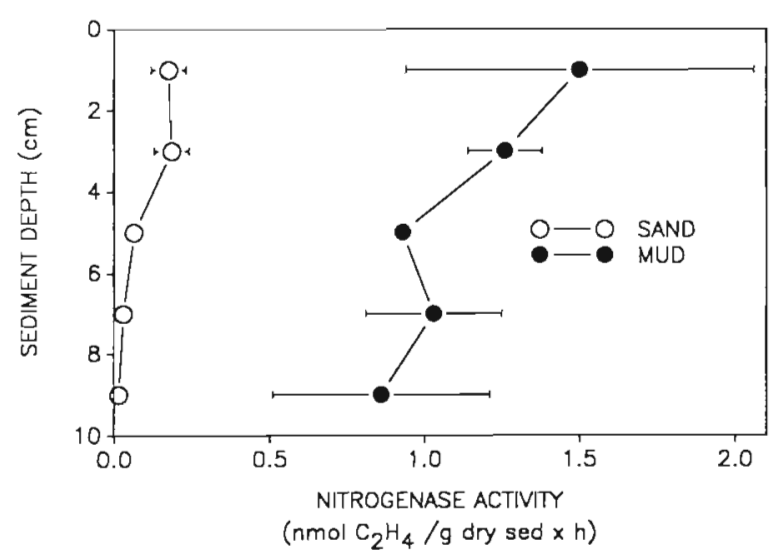

Fig. 3. Sediment depth profiles of nitrogenase activity, under anaerobic conditions. Sandy sediment from Site B2, Puerto Rico. Muddy sediment from Site D2, Puerto Rico

experimental setup: mechanical homogenization vs direct extrusion of cores into flasks as used in our routine assays. Rates of nitrogenase activity were consistently lower in all mechanically (Waring blender; low speed)

Table 3. Comparison of sediment assay methods. Mean rates of nitrogenase activity \pm standard error $(n)$

\begin{tabular}{|c|c|c|}
\hline Site & \multicolumn{2}{|c|}{$\begin{array}{c}\text { Nitrogenase activity } \\
\left.\text { (nmol }[\mathrm{g} \text { dry sed. }]^{-1} \mathrm{~h}^{-1}\right)\end{array}$} \\
\hline Mangrove Bay & $0.181 \pm 0.046(4)$ & $0.296 \pm 0.045$ \\
\hline Devil's Hole & $0.121 \pm 0.005$ & $0.364 \pm 0.099(3)$ \\
\hline Abott's Cliff & $0.196 \pm 0.011$ & $0.355 \pm 0.026(3)^{\circ}$ \\
\hline Outer Reef & $0.031 \pm 0.005(3)$ & $0.764 \pm 0.530$ \\
\hline$\cdot p<0.01$ & & \\
\hline
\end{tabular}

homogenized slurries compared to directly extruded cores (although only significantly lower at the Abott's Cliff site; $\mathrm{p}<0.01$ ) (Table 3). However, the homogenized slurries yielded better replication. Therefore, when homogenization was required (i.e. in amendment experiments), slurrying was accomplished by gently hand mixing with a spatula, which improved replication and did not inhibit rates of nitrogenase activity.

\section{Factors regulating nitrogenase activity}

\section{Oxygen}

In general, there were no consistent or predictable differences between oxic and anoxic incubations among carbonate sediments (Table 4). In organic-rich sediments at Sites D1 and E2, Puerto Rico, rates of anaerobically incubated sediments were significantly higher ( $\mathrm{p}<0.05$, and 0.001 respectively) than aerobically incubated samples. In other organic-rich sites such as Mangrove Bay and Shark Hole, Bermuda, or Pandora Reef, Australia, anaerobically-incubated flasks had average rates of nitrogenase activity slightly higher than aerobically incubated flasks (Fig. 4A). However, lagoonal sediment at the Murray's Anchorage site in Bermuda and off Cockburn Town in San Salvador (the 'Wall'), showed no significant or consistent difference between aerobic or anaerobic incubations. Coarse grained reef sediments in Bermuda $(p<$ 0.005), Bowl Reef, Australia (A, p < 0.001; B, p < 0.005 ), and sandy sediment at Cut Cay, San Salvador ( $p$ $<0.025)$, showed significantly higher rates under aerobic conditions (Fig. 4B; Table 4).

We compared the effect of oxic and anoxic incubations on nitrogenase activity in 3 depth horizons in a sandy sediment from Cut Cay, San Salvador (Fig. 5). Nitrogen fixation under aerobic conditions showed substantial decreases with depth, ranging from $0.40 \mathrm{nmol} \mathrm{C}_{2} \mathrm{H}_{4}$ (g dry sed.) $)^{-1} \mathrm{~h}^{-1}$ in the top $3 \mathrm{~cm}$ to
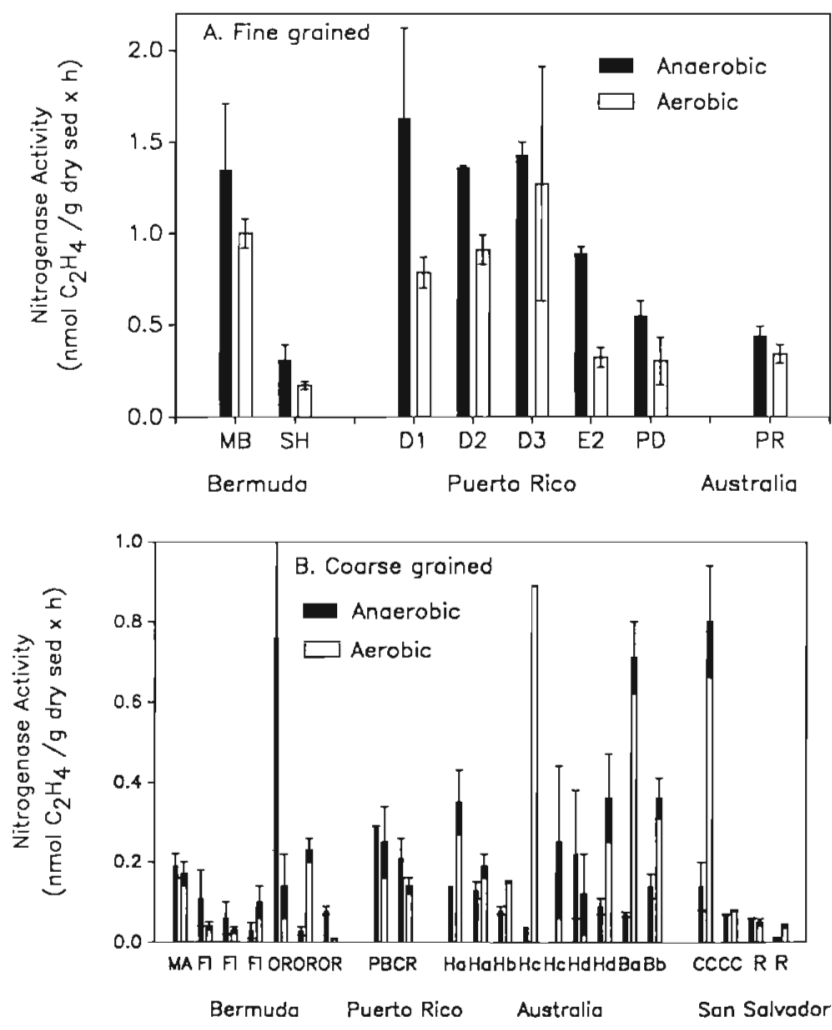

Fig. 4. Effect of anaerobic vs aerobic incubations on rates of nitrogenase activity in (A) fine grained sediments: MB, Mangrove Bay; SH, Shark Hole; D1-PD, points on transect (Fig. 1C); PR = Pandora Reef; and (B) coarse grained sediments: MA, Murray's Anchorage; FI, Flatt's Inlet; OR, Outer Reef; CR, Carabinero Reef; Ha, Hopkinson Reef A, backreef; Hb, Hopkinson Reef B, backreef; Hc, Hopkinson Reef $C_{1}$ sand patch, reef crest; $\mathrm{Hd}$, Hopkinson reef, forereef; Ba, Bowl Reef A; Bb, Bowl Reef B; CC, Cut Cay, sand; R, Cut Cay site reef 
Table 4. Effect of oxygen on nitrogenase activity in carbonate sediments \pm standard error (no. of replicates)

\begin{tabular}{|c|c|c|c|}
\hline \multirow[t]{2}{*}{ Site } & \multirow[t]{2}{*}{ Date } & \multicolumn{2}{|c|}{$\begin{array}{c}\text { Nitrogenase activity } \\
\text { (nmol }[\mathrm{g} \text { dry sed. }]^{-1} \mathrm{~h}^{-1} \text { ) }\end{array}$} \\
\hline & & Anaerobic & Aerobic \\
\hline \multicolumn{4}{|l|}{ Bermuda } \\
\hline \multicolumn{4}{|l|}{ Fine grained } \\
\hline Mangrove Bay & 14 Aug 85 & $1.35 \pm 0.36$ & $1.00 \pm 0.08$ \\
\hline Shark Hole & 20 Aug 85 & $0.31 \pm 0.08$ & $0.17 \pm 0.02$ \\
\hline \multicolumn{4}{|l|}{ Coarse grained } \\
\hline Murray's Anchorage & 28 Aug 85 & $0.19 \pm 0.03$ & $0.17 \pm 0.03$ \\
\hline \multirow[t]{3}{*}{ Flatt's Inlet } & 19 Aug 85 & $0.11 \pm 0.07$ & $0.04 \pm 0.01$ \\
\hline & 24. Aug 85 & $0.06 \pm 0.04$ & $0.03 \pm 0.008$ \\
\hline & 26 Aug 85 & $0.03 \pm 0.02$ & $0.10 \pm 0.04$ \\
\hline \multirow[t]{3}{*}{ Outer Reef } & 28 Aug 85 & $0.76 \pm 0.53$ & $0.14 \pm 0.08$ \\
\hline & 16 Aug 85 & $0.03 \pm 0.01$ & $0.23 \pm 0.03$ \\
\hline & 30 Aug 85 & $0.08 \pm 0.01$ & $0.01 \pm 0.002(3)^{\circ}$ \\
\hline \multicolumn{4}{|l|}{ Puerto Rico } \\
\hline \multicolumn{4}{|l|}{ Fine grained } \\
\hline D1 & 8 Mar 84 & $1.63 \pm 0.49$ & $0.78 \pm 0.085(2)$ \\
\hline $\mathrm{D} 2$ & $8 \mathrm{Mar} 84$ & $1.36 \pm 0.01$ & $0.91 \pm 0.08 \quad(2) \cdots$ \\
\hline D3 & $8 \operatorname{Mar} 84$ & $1.43 \pm 0.07$ & $1.27 \pm 0.64$ \\
\hline E2 & 13 Mar 84 & $0.89 \pm 0.036(4)$ & $0.32 \pm 0.053(4)^{\cdots}$ \\
\hline $\mathrm{PD}$ & 16 Mar 84 & $0.55 \pm 0.08$ & $0.30 \pm 0.13$ \\
\hline \multicolumn{4}{|l|}{ Coarse grained } \\
\hline PB & $16 \operatorname{Mar} 84$ & $0.29 \pm 0.002(2)$ & $0.25 \pm 0.09$ \\
\hline Carabinero Reef & 25 Oct 84 & $0.21 \pm 0.05$ & $0.14 \pm 0.02$ \\
\hline \multicolumn{4}{|l|}{ Australia } \\
\hline \multicolumn{4}{|l|}{ Fine grained } \\
\hline Pandora Reef & 12 Nov 85 & $0.44 \pm 0.05$ & $0.34 \pm 0.05$ \\
\hline \multicolumn{4}{|l|}{ Coarse grained } \\
\hline Hopkinson Reef, A & 8 Nov 85 & 0.14 & $0.35 \pm 0.03$ \\
\hline & $17 \mathrm{Dec} 85$ & $0.13 \pm 0.02$ & $0.19 \pm 0.03$ \\
\hline Hopkinson Reef, B & 17 Dec 85 & $0.08 \pm 0.01$ & $0.15 \pm 0.00$ \\
\hline \multirow[t]{2}{*}{ Hopkinson Reef, C } & $18 \operatorname{Dec} 85$ & 0.04 & 0.89 \\
\hline & 21 Dec 85 & - & $0.25 \pm 0.19$ \\
\hline \multirow[t]{2}{*}{ Hopkinson Reef, D } & 16 Dec 85 & $0.22 \pm 0.16$ & $0.12 \pm 0.10$ \\
\hline & $18 \operatorname{Dec} 85$ & $0.09 \pm 0.02$ & $0.36 \pm 0.11$ \\
\hline Bowl Reef, A & $13 \operatorname{Dec} 85$ & $0.07 \pm 0.006(4)$ & $0.77 \pm 0.09$ \\
\hline Bowl Reef, B & 12 Dec 85 & $0.14 \pm 0.03$ & $0.36 \pm 0.05$ \\
\hline \multirow{2}{*}{\multicolumn{4}{|c|}{$\begin{array}{l}\text { San Salvador } \\
\text { Coarse grained }\end{array}$}} \\
\hline & & & \\
\hline \multirow{2}{*}{$\begin{array}{l}\text { Cut Cay } \\
\text { Curse gramea }\end{array}$} & 11 Jul 86 & $0.14 \pm 0.06$ & $0.80 \pm 0.14$ \\
\hline & 13 Jul 86 & $0.07 \pm 0.001(2)$ & $0.08 \pm 0.002(2)$ \\
\hline \multirow{2}{*}{ Reef } & 14 Jul 86 & $0.06 \pm 0.001(2)$ & $0.05 \pm 0.008(2)$ \\
\hline & 17 Jul 86 & $0.01+0.002(2)$ & $0.04 \pm 0.005(2)$ \\
\hline
\end{tabular}

$0.008 \mathrm{nmol}(\mathrm{g} \text { dry sed. })^{-1} \mathrm{~h}^{-1}$ at a depth of 6 to $8 \mathrm{~cm}$. Anoxic incubations of the same sediment showed a more uniform distribution of rates with depth, ranging from $0.14 \mathrm{nmol}$ (g dry sed.) $)^{-1} \mathrm{~h}^{-1}$ in the top 0 to $3 \mathrm{~cm}$ to $0.044 \mathrm{nmol}$ (g dry sed.) $)^{-1} \mathrm{~h}^{-1}$ in the 6 to $8 \mathrm{~cm}$ horizon.

Results from experiments performed on 3 separate occasions at the same coarse grain site on the outer reef in Bermuda indicated that the nature of the $\mathrm{N}_{2}$ fixing bacterial population may be shifted by physical disturbances and sediment aeration (Fig. 6). The first sampling on July 28, 1985 was after a very calm period when algal mats were observed on the surface of the sediment. On this day, the anaerobic rate was considerably higher than the aerobic incubation (although not significant at the $p<0.05$ level). The edge of tropical storm 'Claudette' moved through on August 12 and 


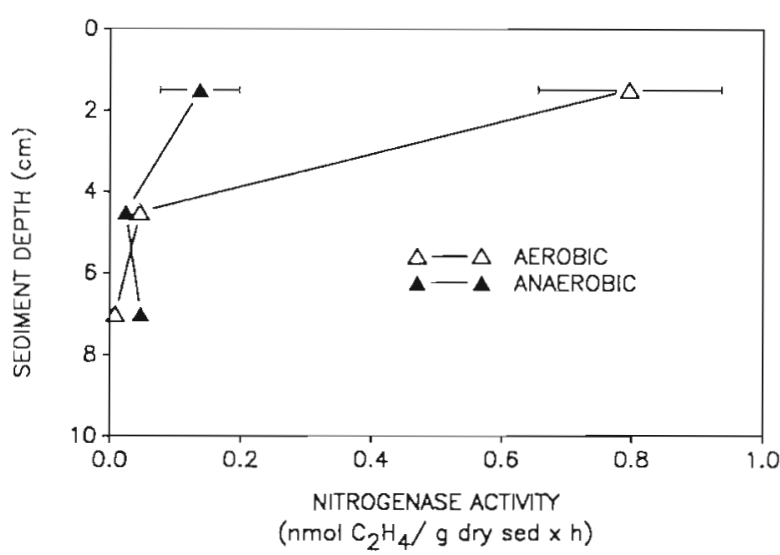

Fig. 5. Nitrogenase activity with depth in sandy sediment trom Cut Cay, San Salvador under aerobic and anaerabic conditions. Error bars indicate \pm standard error; $n=3$

strong winds persisted for several days. Samples were taken again $4 \mathrm{~d}$ after the storm on August 16. The lack of algal mats and the presence of sand ripples indicated that the sediment had been perturbed. Rates of aerobic nitrogenase activity on this day were significantly $(p<0.005)$ greater than the anaerobic incubation. Two weeks later, anaerobic rates, still depressed relative to the first sampling, were once again significantly $(p<0.005)$ higher than aerobic rates.

\section{Ammonium}

Pore water $\mathrm{NH}_{4}^{+}$concentrations ranged from about $1 u M$ to values in excess of $0.5 \mathrm{mM}$ (Tables 1 and 2). However, there was no significant correlation between pore-water $\mathrm{NH}_{4}^{+}$concentrations from the top 0 to $5 \mathrm{~cm}$ of sediment, and rates of nitrogenase activity for the

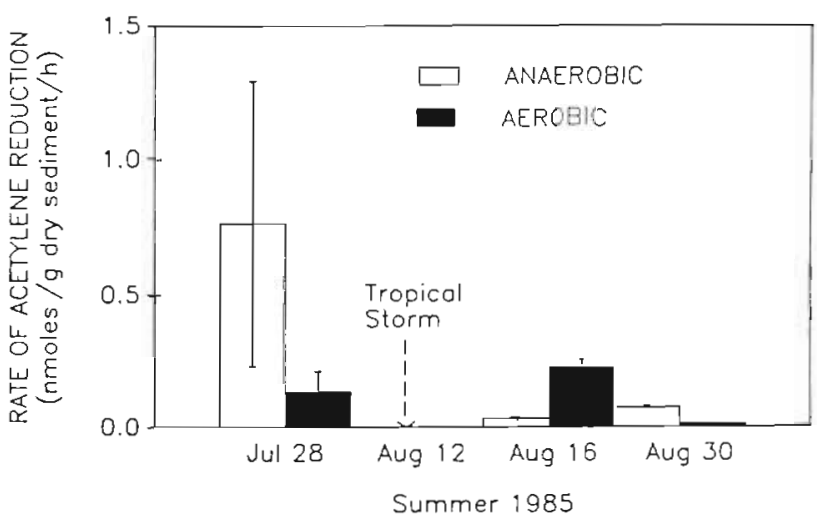

Fig. 6. Nitrogenase activity (acetylene reduction) at the Outer Reef site, Bermuda, before and after tropical storm 'Claudette', Aug 12, 1985. Error bars indicate \pm standard error; $\mathrm{n}=3$

sites where ammonium data was available. To further investigate the effect of $\mathrm{NH}_{4}^{+}$on nitrogenase activity, additions of $\mathrm{NH}_{4} \mathrm{Cl}$ ranging from $100 \mu \mathrm{M}$ to $5 \mathrm{mM}$ were made to sediments and their effect on nitrogenase activity observed (Table 5). Additions of 100 to $500 \mu \mathrm{M}$ did not significantly inhibit nitrogenase activity at any of the sites. However, additions of $1 \mathrm{mM}$ significantly $(p<0.001)$ reduced nitrogenase activity at the Outer Reef, Bermuda site and $5 \mathrm{mM}$ reduced nitrogenase activity by 75 to $80 \%(p<0.005)$ at the Devil's Hole site in Bermuda. Devil's Hole was also the only site in Bermuda in which additions of MSX $\left(10 \mathrm{mg} \mathrm{ml}^{-1}\right)$ showed any stimulation of rates (although not significant at $p<0.05$ level) (Table 6). However, MSX appeared to reduce nitrogenase activity at all other sites in Bermuda and San Salvador. Therefore, at in situ concentrations in the majority of these sediments, $\mathrm{NH}_{4}^{+}$ does not inhibit nitrogenase activity.

Table 5. Anaerobic rates of nitrogenase activity ( $\mathrm{nmol}$ ethylene $\mathrm{g}^{-1} \mathrm{~h}^{-1}$; mean \pm standard error) with ammonium additions $(\mathrm{n}=3$ )

\begin{tabular}{|c|c|c|c|c|c|}
\hline \multirow[t]{2}{*}{ Sites } & \multicolumn{5}{|c|}{ Ammonium additions } \\
\hline & Control & $100 \mu M$ & $500 \mu M$ & $1 \mathrm{~m} M$ & $5 \mathrm{mM}$ \\
\hline \multicolumn{6}{|l|}{ Bermuda } \\
\hline Mangrove Bay & $0.242 \pm 0.013$ & & & \multirow[t]{2}{*}{$0.254 \pm 0.065$} & \\
\hline Devil's Hole & $0.364 \pm 0.099$ & & & & $0.079 \pm 0.003^{\circ}$ \\
\hline Muray's Anchorage & $0.079 \pm 0.040$ & $0.070 \pm 0.006$ & & & \\
\hline Flatt's Inlet & $0.105 \pm 0.066$ & & & & $0.044 \pm 0.011$ \\
\hline Shark Hole & $0.312 \pm 0.080$ & $0.250 \pm 0.046$ & & & \\
\hline \multirow[t]{3}{*}{ Outer Reef } & $0.026 \pm 0.015$ & & & \multirow[t]{3}{*}{$0.015 \pm 0.004^{\cdots}$} & \\
\hline & $0.034 \pm 0.005$ & & $0.030 \pm 0.007$ & & \\
\hline & $0.076 \pm 0.006$ & $0.090 \pm 0.009$ & & & \\
\hline \multicolumn{6}{|l|}{ San Salvador } \\
\hline Cut Cay sand & $0.068 \pm 0.001$ & & $0.058 \pm 0.026$ & & \\
\hline Cockburn Town 'Wall' & $0.087 \pm 0.013$ & & $0.081 \pm 0.008$ & & \\
\hline \multicolumn{6}{|l|}{$\cdot p<0.005 ; \cdots p<0.001$} \\
\hline
\end{tabular}


Table 6. Rates of anaerobic nitrogenase activity with additions of $10 \mathrm{mg} \mathrm{ml}^{-1}$ L-methionine-DL-sulfoximine (MSX). Mean \pm standard error. $(n)=3$, unless otherwise noted

\begin{tabular}{|c|c|c|}
\hline \multirow[t]{2}{*}{ Sites } & \multicolumn{2}{|c|}{$\begin{array}{c}\text { Nitrogenase activity } \\
\text { (nmol [g dry sed. }]^{-1} \mathrm{~h}^{-1} \text { ) }\end{array}$} \\
\hline & Control & $+\mathrm{MSX}$ \\
\hline \multicolumn{3}{|l|}{ Bermuda } \\
\hline Mangrove Bay & $\begin{array}{l}0.152 \pm 0.090(4) \\
1.190 \pm 0.750\end{array}$ & $0.030 \pm 0.005(4)$ \\
\hline Devil's Hole & $0.042 \pm 0.018$ & $0.070 \pm 0.002$ \\
\hline Abott's Cliff & $0.158 \pm 0.040$ & $0.093 \pm 0.029$ \\
\hline $\begin{array}{l}\text { Murray's } \\
\text { Anchorage }\end{array}$ & $0.079 \pm 0.040$ & $0.049 \pm 0.010$ \\
\hline Shark Hole & $0.123 \pm 0.014$ & $0.130 \pm 0.015$ \\
\hline \multicolumn{3}{|l|}{ San Salvador } \\
\hline Cut Cay (sand) & $0.093 \pm 0.002$ & $0.071 \pm 0.002$ \\
\hline $\begin{array}{l}\text { Cut Cay } \\
\text { (Syringodium) }\end{array}$ & $0.187 \pm 0.040(2)$ & $0.296 \pm 0.033(2)$ \\
\hline Wall site & $0.087 \pm 0.013$ & $0.076 \pm 0.013$ \\
\hline Reef & $0.099 \pm 0.060$ & $0.026 \pm 0.013$ \\
\hline
\end{tabular}

\section{Organic substrate availability}

While there was no significant relationship between nitrogenase activity and organic content among all stations sampled, there was a strong positive correlation $\left(r^{2}=0.78\right)$ when samples from eutrophic sites at La Parguera, Puerto Rico, were ignored. Among sites sampled in Bermuda and Australia, even higher correlations were noted $\left(\mathrm{r}^{2}=0.86\right.$ and 0.96 , respectively). In general, inshore sites or nearshore sites had higher percent organic content and nitrogenase activity than offshore sites.

Additions of readily oxidizable organic substrates (glucose and mannitol) of $100 \mu \mathrm{M}$ or more significantly stimulated rates of nitrogenase activity at all sites investigated (Table 7). The extent of stimulation was concentration-dependent. Addition of $10 \mu \mathrm{M}$ mannitol at the reef site in San Salvador, while not significant at the $\mathrm{p}<0.05$ level, stimulated rates 1.3 times that of the control rate. Addition of $100 \mu \mathrm{M}$ mannitol yielded a rate 13 times $(p<0.025)$ the control rate. Addition of $3 \mathrm{mM}$ mannitol to coarse grained sediment from the Outer Reef in Bermuda increased rates ca 400 times the control rate $(\mathrm{p} \ll 0.005)$.

\section{DISCUSSION}

The trend towards higher rates of nitrogenase activity in areas of relatively high ambient $\mathrm{NH}_{4}^{+}$concentration is enigmatic as it has been shown that the presence of high $\mathrm{NH}_{4}^{+}$represses $\mathrm{N}_{2}$ fixation (Capone 1988). However, these same environments are generally also zones of higher organic content and lower $\mathrm{O}_{2}$ concentration. Finer grained, organic-rich sediments had the highest rates of nitrogenase activity, and may also have greater numbers of $\mathrm{N}_{2}$-fixing bacteria than coarse sand and coral rubble sediments (Hanson \& Gundersen 1976). Our results indicate that organic substrate availability is a major factor controlling nitrogenase activity in situ in these sediments.

Despite the fact that nitrogenase is highly sensitive to $\mathrm{O}_{2}, \mathrm{~N}_{2}$ fixation can occur under fully oxic to anoxic conditions as organisms have evolved various strategies to protect the enzyme from $\mathrm{O}_{2}$ inactivation (Postgate 1982). The response of $\mathrm{N}_{2}$ fixation to $\mathrm{O}_{2}$ reveals the potential for control by $\mathrm{O}_{2}$ as well as an indication of the physiological makeup of the $\mathrm{N}_{2}$ fixing population. Our experiments provide an integrated assessment over a discrete depth increment of the physiological response of the $\mathrm{N}_{2}$ fixing microbiota to variations in $\mathrm{O}_{2}$. We cannot, however, discern physiological variation within the population, nor any small scale spatial trends in distribution. While we did not obtain $\mathrm{O}_{2}$ profiles, there are sharp gradients of $\mathrm{O}_{2}$ in these sediments (G. King pers, comm.). Organic-rich, highly reducing sediments, not subjected to physical disturbance, might select for strict anaerobes, while aerobes and facultative anaerobes are more likely to predominate in coarse grained or turbulent sedimen-

Table 7. Anaerobic rates of nitrogenase activity (nmol ethylene $\mathrm{g}^{-1} \mathrm{~h}^{-1}$; mean \pm standard error) with organic substrate additions $(\mathrm{n}=3)$

\begin{tabular}{|c|c|c|c|c|c|c|}
\hline Site & Control & $\begin{array}{l}\text { Mannitol } \\
(10 \mu M)\end{array}$ & $\begin{array}{l}\text { Mannitol } \\
(100 \mu M)\end{array}$ & $\begin{array}{l}\text { Mannitol } \\
\text { (3 } \mathrm{mM})\end{array}$ & $\begin{array}{l}\text { Glucose } \\
(10 \mu M)\end{array}$ & $\begin{array}{l}\text { Glucose } \\
(100 \mu M)\end{array}$ \\
\hline \multirow[t]{2}{*}{ San Sal. reef } & $0.058 \pm 0.001$ & & $0.620 \pm 0.108^{\cdots}$ & & & $1.160 \pm 0.120^{\circ}$ \\
\hline & $0.013 \pm 0.002$ & $0.030 \pm 0.015$ & $0.182 \pm 0.103$ & & $0.019 \pm 0.010$ & $0.017 \pm 0.004^{\circ}$ \\
\hline \multirow[t]{2}{*}{ Cut Cay sand } & $\begin{array}{l}0.032 \pm 0.003 \\
0.067 \pm 0.005\end{array}$ & & $\begin{array}{l}0.454 \pm 0.016 \cdots \\
0.324 \pm 0.001 \cdots\end{array}$ & & & $0.558 \pm 0.066^{\circ}$ \\
\hline & $0.054 \pm 0.002$ & & & & $0.067 \pm 0.014$ & $0.584 \pm 0.030^{\circ}$ \\
\hline Bermuda reef & $0.764 \pm 0.006$ & & & $32.92 \pm 3.96 \cdots$ & & \\
\hline
\end{tabular}


tary environments. While we did observe inhibition of nitrogenase activity by $\mathrm{O}_{2}$ in muddy sediments in Puerto Rico, and, conversely, several instances where aerobic rates significantly exceeded anaerobic rates in sandy sediments, these effects were variable and in most cases not significant (Fig. 4).

Aerobes and anaerobes may coexist within the same sediment horizon. Patriquin \& Knowles (1975) pointed out the importance, with respect to $\mathrm{N}_{2}$ fixation, of anaerobic microenvironments maintained by bacterial associations and the physical structure of the skeletal sands. Paerl \& Prufert (1987) noted enhanced nitrogenase activity in the nutrient-enriched micro-environments of suspended or benthic organic detritus and related this enhancement to the $\mathrm{O}_{2}$-free microzones maintained in these aggregates. Therefore, even under seemingly aerobic conditions $\mathrm{N}_{2}$ fixation may be occurring in anaerobic microzones, and the occasional stimulation of nitrogenase activity in sands by anoxic conditions is suggestive of such assemblages.

The difference in rates between anoxic and oxic incubations may be related to the bacterial assemblage in the sediment at that time, as well as a reflection of the recent oxygenation history of the sediments. The variable results obtained at the Outer Reef site in Bermuda on subsequent sampling days may be attributed to disturbance of anaerobic microzones in the sediments that may form during quiescent periods. In such environments, $\mathrm{O}_{2}$ may play a greater role in defining the nature of the $\mathrm{N}_{2}$-fixing population and the extent of their activity.

Inshore sites had higher rates of nitrogenase activity and, enigmatically, the highest average $\mathrm{NH}_{4}^{+}$concentrations. These sites were also characterized by higher average organic content and, probably, higher bacterial numbers (Hanson \& Gundersen 1976). However, pore-water $\mathrm{NH}_{4}^{+}$values at most remote sites were well below $100 \mu \mathrm{M}$; this is below, or just at, the thresholds reported for nitrogenase repression. In salt marsh sediments, Carpenter et al. (1978) found the threshold for repression of nitrogenase activity to be $100 \mu \mathrm{M} \mathrm{NH}_{4}^{+}$ and Teal et al. (1979) found the threshold of repression to be $200 \mu \mathrm{MNH} \mathrm{NH}_{4}^{+}$. Similar results have been found in estuarine and carbonate muds (Capone 1988). In carbonate sediments of Puerto Rico, Nieves \& Corredor (1987) found evidence indicating 2 distinct populations of $\mathrm{N}_{2}$ fixers; one group susceptible and the other not susceptible to ammonium inhibition above $200 \mu \mathrm{M}$.

In our experimental studies, the only significant

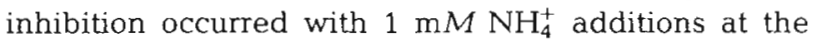
Outer Reef, Bermuda ( $\mathrm{p}<0.001)$ and $5 \mathrm{mM}$ additions at Devil's Hole, Bermuda ( $p<0.005)$. The Outer Reef site had very low porewater ammonium $(3 \mu M)$, and $1 \mathrm{mM}$ was an extreme increase over ambient levels of $\mathrm{NH}_{4}^{+}$. Devil's Hole sediments had a porewater ammonium concentration of $37 \mu M$ in the upper few centimeters and concentrations of up to $300 \mu \mathrm{MNH}_{4}^{+}$at a depth of 5 $\mathrm{cm}$ in the sediment have been found at this site (Thorenston \& Mackenzie 1974). Devil's Hole was also the only site where any increase of rates was obtained with additions of MSX. This suggests that $\mathrm{NH}_{4}^{+}$may repress rates of nitrogenase activity in situ at Devil's Hole.

Nitrogen fixation is energetically demanding and, therefore, heterotrophic diazatrophs may have a greater need than non- $\mathrm{N}_{2}$-fixing bacteria to oxidize organic substrates. Of those determinants investigated in our study, organic substrate availability was the most important factor controlling rates of nitrogenase activity. Rates of nitrogenase activity were highest in sediments with highest organic content, despite high $\mathrm{NH}_{4}^{+}$ concentrations at the same sites. Furthermore, additions of the organic substrates, mannitol and glucose, showed a concentration-dependent stimulation of nitrogenase activity. The availability of readily metabolizable organic compounds may play a second role in heterotrophic $\mathrm{N}_{2}$ fixation, by promoting areas of localized oxygen consumption where reduced oxygen microzones can exist (Paerl \& Prufert 1987, Paerl et al. 1987).

To calculate estimates of nitrogen fixation from rates of nitrogenase activity, a ratio of $3: 1$ moles $\mathrm{C}_{2} \mathrm{H}_{2}$ reduced per mole $\mathrm{N}_{2}$ is usually used, based on the relative number of electrons transferred in each reaction. However, in natural populations of bacteria, ratios are closer to $4: 1$, due to $\mathrm{H}_{2}$ production associated with $\mathrm{N}_{2}$ fixation but not $\mathrm{C}_{2} \mathrm{H}_{2}$ reduction (Postgate 1982). We did not perform ${ }^{15} \mathrm{~N}_{2}$ calibration in our studies; however, reported ratios for similar carbonate sediments range from 0.5 to 10.5 (Capone 1983). Therefore, we chose to use $4: 1$ as an average value. Using a sediment density of $0.50 \mathrm{~g}$ dry $\mathrm{wt} \mathrm{cm}^{-3}$ for sands and $0.30 \mathrm{~g}$ dry wt $\mathrm{cm}^{-3}$ for finer grained muds, and assuming a constant rate of nitrogenase activity to $6 \mathrm{~cm}$, rates of nitrogenase activity in $\mathrm{nmol} \mathrm{C}_{2} \mathrm{H}_{2}(\mathrm{~g} \text { dry sed.) })^{-1} \mathrm{~h}^{-1}$ were converted to rates of $\mathrm{N}_{2}$ fixation in terms of $\mathrm{mg} \mathrm{N}$ $\mathrm{m}^{-2} \mathrm{~d}^{-1}$. Areal rates among all sites ranged from 0.57 to $5.2 \mathrm{mg} \mathrm{N} \mathrm{m}^{-2} \mathrm{~d}^{-1}$ for the muds, and from 0.07 to $3.9 \mathrm{mg}$ $\mathrm{N} \mathrm{m}^{-2} \mathrm{~d}^{-1}$ in sands (Table 8 ).

In an earlier study near Magnetic Island, Australia, adjacent to Townsville, Crossland \& Barnes (1976) noted much higher $\mathrm{N}_{2}$ fixation rates than ours in muds, with values at the high end of and above our observed range in sands. Similarly, Hanson \& Gundersen (1976) also found relatively higher rates, compared to our range, in the eutrophic sediments of Kaneohe Bay, Hawaii (Table 8). However, except for the samples from La Parguera and inshore sites around Bermuda, most of our studies focused on oligotrophic sites with less nutrient and organic inputs. 
Table 8. Comparison of $\mathrm{N}_{2}$ fixation estimates in shallow carbonate sediments

\begin{tabular}{|lccc|}
\hline Site & \multicolumn{2}{c}{$\begin{array}{c}\text { Nitrogen fixation } \\
\left(\mathrm{mg} \mathrm{N} \mathrm{m}^{-2} \mathrm{~d}^{-1}\right) \\
\text { Coarse grained }\end{array}$} & Source \\
\hline Bermuda & Fine grained & $0.13-3.85$ & This study \\
Puerto Rico & $0.57-4.08$ & $0.71-1.46$ & This study \\
San Salvador & $1.66-5.17$ & $0.07-0.69$ & This study \\
Australia & - & $0.20-1.41$ & This study \\
Australia & 1.33 & $3.52-5.0$ & Crossland \& Barnes (1976) \\
Hawaii & 28 & $1.30-5.8$ & Hanson \& Gundersen (1976) \\
Barbados & - & 0.9 & Patriquin \& Knowles (1972) \\
\hline
\end{tabular}

Capone \& Carpenter (1982) estimated that $\mathrm{N}_{2}$ fixation in coral reef environments could contribute $25 \mathrm{~g} \mathrm{~N}$ $\mathrm{m}^{-2} \mathrm{yr}^{-1}$ to the annual input of combined nitrogen to the global nitrogen cycle, accounting for $14 \%$ of recognized oceanic inputs. This value did not, however, include heterotrophic $\mathrm{N}_{2}$ fixation in the sediments. On an areal basis, heterotrophic $\mathrm{N}_{2}$ fixation in carbonate sediments could account for 0.03 to $1.9 \mathrm{~g} \mathrm{~N} \mathrm{~m}^{-2} \mathrm{yr}^{-1}$, or about 0.1 to $8 \%$ of the total rate for the reef proper. We suggest that an areally weighted and integrated extrapolation, considering the extensive shallow sedimentary non-reef environments, would reveal that the actual amount of $\mathrm{N}_{2}$ contributed locally (as well as to the global nitrogen cycle) may be greater from coral reef sediments than the reef proper. In this respect, Hanson \& Gundersen (1977) noted that encrusted coral cover (sites of the most intense photoautotrophic $\mathrm{N}_{2}$ fixation) in Kaneohe Bay covered about 1 ha $\left(0.01 \mathrm{~km}^{2}\right)$ and accounted for an annual total of $0.8 \times 10^{3} \mathrm{~kg} \mathrm{~N}$. The minimum area of sediment cover in the bay was 46 $\mathrm{km}^{2}$ which accounted for a total of $28 \times 10^{3} \mathrm{~kg} \mathrm{~N} \mathrm{yr}^{-1}$, or at least 35 times as much as the cyanobacterial activity.

The relatively low ambient concentrations of $\mathrm{NH}_{4}^{+}$in shallow carbonate sediments (Corredor \& Capone 1985), compared to shallow sediments in temperate zones, may allow heterotrophic $\mathrm{N}_{2}$ fixation to be a more prominent component of the sedimentary nitrogen cycle in tropical environments than in temperate regions (Seitzinger \& Garber 1987. Capone 1988) In order to place in situ fixation in the context of nitrogen cycling in sediments, we compared the extrapolated rate of $\mathrm{N}_{2}$ fixation to observed levels of $\mathrm{NH}_{4}^{+}$over the same depth range for those stations and times that the $\mathrm{NH}_{4}^{+}$data were available. The percent of porewater $\mathrm{NH}_{4}^{+}$that could be contributed by benthic heterotrophic $\mathrm{N}_{2}$ fixing bacteria per day was estimated to be between 1.2 and $11 \%$ for muds and from 3 to $70 \%$ for sands. Turnover time based on $\mathrm{N}_{2}$ fixation was calculated to be 1.4 to $86 \mathrm{~d}$. These values emphasize that benthic heterotrophic $\mathrm{N}_{2}$ fixers can contribute substantially to the pool of $\mathrm{NH}_{4}^{+}$in the sediments and sedimentary $\mathrm{N}_{2}$ fixation should be taken into account when establishing nitrogen balances in these environments.

Other components of nitrogen cycling such as ammonification and dissimilatory nitrate reduction may contribute a larger fraction to the sedimentary $\mathrm{NH}_{4}^{+}$pool and, therefore, the actual turnover rates in reef environments are probably higher. Williams et al. (1985) estimated ammonification in homogenized reef sediments of St. Croix (U.S. Virgin Islands) and found an average net $\mathrm{NH}_{4}^{+}$production rate of $70 \mu \mathrm{mol}\left(\mathrm{l} \mathrm{sed}\right.$.) ${ }^{-1} \mathrm{~d}^{-1}$ (ca 60 $\mathrm{mg} \mathrm{N} \mathrm{m}{ }^{-2} \mathrm{~d}^{-1}$ ) which, for $\mathrm{NH}_{4}^{+}$concentrations from 0 to $79 \mu \mathrm{M}$, could account for turnover times of up to $0.74 \mathrm{~d}-$ substantially shorter than turnover times that could be attributed to $\mathrm{N}_{2}$ fixation. Capone et al. (unpubl.) performed ammonification experiments on intact cores of medium to coarse grained sediments from Bowl and Hopkinson Reefs, Australia and found much lower rates ( 2 to $9 \mathrm{mg} \mathrm{N} \mathrm{m}^{-2} \mathrm{~d}^{-1}$ ). Similarly, Corredor \& Morell (1985) estimated diffusion-driven ammonium fluxes of 3 to $5 \mathrm{mg} \mathrm{N} \mathrm{m}^{-2} \mathrm{~d}^{-1}$ from reef sediments. Relative to these lower estimates, $\mathrm{N}_{2}$ fixation takes on a much greater importance. It should be noted that the $\mathrm{NH}_{4}^{+}$derived from ammonification represents recycled nitrogen when considered from the view of the sediment-water system, while $\mathrm{NH}_{4}^{+}$arising from $\mathrm{N}_{2}$ fixation constitutes de novo input. Given minimal external nitrogen inputs in the oligotrophic tropical waters, this new input could be of critical importance in the maintenance of reef productivity.

\section{SUMMARY AND CONCLUSIONS}

(1) The effect of oxygen on nitrogenase activity was highly variable and not consistent among sediment types. Nitrogenase activity appeared to be influenced by physical disturbance of the sediment, possibly due to sediment aeration. 
(2) The absence of (a) an inverse correlation between porewater $\mathrm{NH}_{4}^{+}$concentration on nitrogenase activity, (b) an effect of $\mathrm{NH}_{4}^{+}$additions on nitrogenase activity at most sites, or (c) stimulation by the derepressor MSX, suggests that $\mathrm{NH}_{4}^{+}$is not a significant factor regulating in situ $\mathrm{N}_{2}$ fixation in the carbonate sediments investigated

(3) Nitrogenase activity increased significantly with organic substrate availability. A correlation between rates of nitrogenase activity and total organic content of sediments was observed at all experimental sites. Additions of organic substrates (mannitol and glucose) produced significant, concentration-dependent stimulation of rates of nitrogenase activity. Therefore, organic substrate availability appears to be the most significant factor controlling $\mathrm{N}_{2}$ fixation in the carbonate sediments investigated.

(4) Based on our experiments, $N_{2}$ fixation may account for an appreciable fraction of the nitrogen input within these sediments, and may also account for substantial overall inputs in oligotrophic tropical waters.

Acknowledgements. The authors thank W. C. Dennison for help in the field and for critically reviewing this manuscript and S. E. Dunham, S. R. Smith, L. Duguay, J. Corredor, and J. Morell for logistical help and nutrient analyses. We also thank colleagues at the various laboratory facilities used including: the Bermuda Biological Station, the Australian Institute of Marine Sciences, field stations at La Parguera, Puerto Rico (Department of Marine Sciences, University of Puerto Rico, Mayaguez), and the College Center of the Finger Lakes in San Salvador; also, the Masters and crew aboard the RV 'Calanus' in San Salvador, the RV 'Pezmar' in Puerto Rico, and the RV 'Sirius' and RV 'Lady Basten' on the Great Barrier Reef. This research was supported by National Science Foundation Grants OCE-85-18491, OCE-84-17595, OCE-8742088, INT83-17747; a Sydney L. Wright Fellowship from the Bermuda Biological Station; NY Sea Grant Institute Project R/M-50, and the Marine Sciences Research Center. Thanks also to Hans Paerl and 2 anonymous reviewers for very helpful comments on this manuscript.

\section{LITERATURE CITED}

Capone, D. G. (1982). Nitrogen fixation (acetylene reduction) by rhizosphere sediments of the eelgrass, Zostera marina L. Mar. Ecol. Prog. Ser. 10:67-75

Capone, D. G. (1983). Benthic nitrogen fixation. In: Carpenter, E. J., Capone, D. C. (eds.) Nitrogen in the marine environment. Academic Press, New York, p. 105-137

Capone, D. G. (1988). Benthic nitrogen fixation: microbiology, physiology and ecology. In: Blackburn, T. H., Sorensen, J., Roswall, T. (eds.) Nitrogen cycling in marine coastal environments. SCOPE Series. J. Wiley \& Sons, New York, p. $85-123$

Capone, D. G., Carpenter, E. J. (1982). Nitrogen fixation in the marine environment. Science 217: 1140-1142

Carpenter, E. J., Van Raalte, C. D., Valiela, I. (1978). Nitrogen. fixation by algae in a Massachusetts salt marsh. Limnol. Oceanogr. 23: 318-327
Corredor, J. E., Capone, D. G. (1985). Studies on nitrogen diagenesis in coral reef sands. Proc. Fifth Int. Coral Reef Symp. 3: 395-399

Corredor, J. E., Morell, J. (1985). Inorganic nitrogen in coral reef sediments. Mar Chem. 16: 379-384

Crossland, C. J., Barnes, D. J. (1976). Acetylene reduction by coral skeletons. Limnol. Oceanogr. 21: 153-156

D'Elia, C. F., Wiebe,W. J. (in press). Biogeochemical nutrient cycles in coral reef ecosystems. In: Dubinsky, S. (ed.) Coral reef ecosystems. Ecosystems of the World Series. Elsevier Science Publishers, Amsterdam

Gaudette, H. E., Flight, W. R., Toner, L., Folger, D. W (1974). An inexpensive titration method for the determination of organic carbon in recent sediments. J. sedim. Petrol. 44: $249-253$

Gordon, J. K., Brill, W. J. (1974). Derepression of nitrogenase synthesis in the presence of excess $\mathrm{NH}_{4}^{+}$. Biochem. Biophys. Res. Comm. 59: 967-971

Hanson, R. B., Gundersen, K. R. (1976). Bacterial nitrogen fixation in a polluted coral reef flat ecosystem, Kaneohe Bay, Oahu, Hawaiian Islands. Pacif. Sci. 30: 385-393

Hanson, R. B., Gundersen, K. (1977). Relationship between nitrogen fixation (acetylene reduction) and the $C: N$ ratio in a polluted coral reef ecosystem, Kaneohe Bay, Hawaii. Estuar. coast. mar. Sci. 5: 437-444

Hardy, R. W. F., Holsten, R. D., Jackson, E. K., Burns, R. C. (1968). The acetylene-ethylene assay for $N_{2}$ fixation: laboratory and field evaluation. Plant Physiol. 43: $1185-1207$

Howarth, R. W., Marino, R., Lane, J., Cole, J. J. (1988), Nitrogen fixation in freshwater, estuarine, and marine ecosystems. 1. Rates and importance. Limnol. Oceanogr 33: 669-687

Nieves, F. A., Corredor, J. E. (1987). Gradientes de fijacion de nitrogeno en los sedimentos marinos asociados a arrecifes coralinos en el suroeste de Puerto Rico. An. Inst. Inv. Mar. Punta de Betin, Santa Marta, Colombia, 17: 27-37

Paerl, H. W., Crocker, K. M., Prufert, L. E. (1987). Limitation of $\mathrm{N}_{2}$ fixation in coastal marine waters: relative importance of molybdenum, iron, phosphorus, and organic matter availability. Limnol. Oceanogr. 32: 525-536

Paerl, H. W., Prufert, L. E. (1987). Oxygen-poor microzones as potential sites of microbial $\mathrm{N}_{2}$ fixation in nitrogen-depleted aerobic marine waters. Appl. environ. Microbiol. 53: 1078-1087

Patriquin, D. G., Knowles, R. (1972). Nitrogen fixation in the rhizosphere of marine angiosperms. Mar. Biol. 16: 49-62

Patriquin, D. G., Knowles, R. (1975). Effects of oxygen, mannitol, and ammonium concentrations on nitrogenase $\left(\mathrm{C}_{2} \mathrm{H}_{2}\right)$ activity in a marine skeletal carbonate sand. Mar. Biol. 32: 49-62

Postgate, J. R. (1982). The fundamentals of nitrogen fixation. Cambridge University Press, New York

Seitzinger, S. P., Garber, J. H. (1987). Nitrogen fixation and ${ }^{15} \mathrm{~N}_{2}$ calibration of the acetylene reduction assay in coastal marine sediments. Mar. Ecol. Prog. Ser. 37: 65-73

Short, F. T., Davis, M. W., Gibson, R. A., Zimmerman, C. F (1985). Evidence for phosphorus limitation in carbonate sediments of the seagrass Syringodium filiforme. Estuar. coast. Shelf Sci. 20: 419-430

Smith, S. V. (1984). Phosphorus versus nitrogen limitation in the marine environment. Limnol. Oceanogr. 29: 1149-1160

Sokal, R. R., Rohlf, F. J. (1981). Biometry. W H. Freeman Co., New York

Stewart, W. P. D., Fitzgerald, G. P., Burris, R. H. (1967). In situ studies on $\mathrm{N}_{2}$ fixation using the acetylene reduction technique. Proc. natn. Acad. Sci. U.S. 58: 2071-2078 
Strickland, J. D. H., Parsons, T R. (1972). A practical handbook of seawater analysis. Bull. Fish. Res. Bd Can. 167

Teal, T M., Valiela, I., Berlo, D. (1979). Nitrogen fixation by rhizosphere and free-living bacteria in salt marsh sediments. Limol. Oceanogr. 24: 126-132

Thorenston, D. C., Mackenzie, F. T. (1974). Time variability of pore water chemistry in recent carbonate sediments, Devil's Hole, Harrington Sound, Bermuda. Geochim. Cosmochim. Acta 38: 1-19

This article was presented by Professor K. R. Tenore, Solomons, Maryland, USA
Williams, S. L., Yarish, S. M., Gill, I. P. (1985). Ammonium distributions, production, and efflux from backreef sediments, St. Croix, U.S. Virgin Islands. Mar. Ecol. Prog. Ser. 24: $57-64$

Yoch, D. C., Whiting, G. J. (1986). Evidence for $\mathrm{NH}_{4}^{+}$switch-off regulation of nitrogenase activity by bacteria in salt marsh sediments and roots of the grass Spartina alterniflora. Appl. environ. Microbiol. 51: 143-149

Manuscript first received: November 2, 1988

Revised version accepted: May 26, 1989 